\title{
4.1
}

\section{Digital Hybrids - Between Tool and Methods: An Introduction and Overview}

\author{
Konstantinos Ioannidis ${ }^{1(\bowtie)}\left(\right.$ (D) and Carlos Smaniotto Costa $^{2}($ D \\ 1 aaiko arkitekter, Oslo, Norway \\ konionn@aaiko.no \\ ${ }^{2}$ Universidade Lusófona, Interdisciplinary Research Centre for Education \\ and Development CeiED, Lisbon, Portugal \\ smaniotto.costa@ulusofona.pt
}

\begin{abstract}
Now, with new digital intersections, digital hybrids, digital combinations, the risk is that [space] is simply incapable of thinking of its entire repertoire ... This is the moment where elements [of space] that have never spoken or never listened are turning into communicating elements. Rem Koolhaas, quoted from Winston (2014).
\end{abstract}

\section{Introduction: The Blur of the Physical Space}

If last century's conception of open public space was understood as a performance stage where individuals could negotiate and establish relationships not only amongst them but also with elements of spatial manifestation demonstrating the significance of some visual, most times, qualities, a contemporary observer will probably not make this link. Today's conception of outdoor space cannot escape from being injected inside the relatively new scene of simulation which constantly changes the way people experience the given inputs. This scene has dramatically modified the series of our somehow culturally pre-structured views or habitual frames of reference about what is real, virtual, represented, perceived, fragmented or holistically experienced.

In the above introductory quote by Koolhaas, the provocation implied by the use of the words "incapability of thinking", as opposed to "turning into communicating elements", is largely related to the emerging conditions for the public open space in the contemporary post-digital era and the neo-analogue mode of outdoor lifestyle. However, in what follows in these chapters, our point is not just to stress a particular trend in the way ICT transform, rather inevitably, our outdoor living patterns, but to argue that such an incapability opens up the opportunity to challenge the human aspect beyond the digital, both spatially and conceptually. As of this writing, locomotional participation, agency and interactivity between the user and the digitally displayed material projected on a mobile device seem to appear more and more often as critical introductory elements of an evolved method of (re)connecting man, space and information. Mitchell (2004), in his influential Me ++: The Cyborg Self and the Networked City, argues that this is a method of completing "a long project of seamlessly integrating our mobile biological bodies with globally extended systems of nodes and linkages". For this session, these are not only simulation techniques that are called to somehow respond to the digital growth of our analogue surroundings. They also offer to a method 
of decrypting that reassigns meanings and referents between man and space, between digital and physical landscapes. In this respect, the proposed method explores different mechanisms of awareness: tropes that manifest themselves as systems of hyperconnectivity in Mitchell's (2014:58) sense and as an arsenal of emerging concepts like those of blended spaces, digital hybrids, user empowerment or enhanced connectivism.

Some of the functional and methodological aspects out of these concepts are highlighted in the following chapters. Within them, the term digital hybrids makes it possible for the idea of enhancing places to detach from the "functional code" and refer to the process whereby research techniques and methodologies can standoff from the mute digitization of outdoor spatial experience. It could be argued that it is in book part thanks to multidisciplinary collaborative research, like the CyberParks TU1306 Project, that alternative forays for digitally mediated agency are currently explored. Moreover, we can argue that the detachment from the functionality of the code and the shift in thinking with the digital is somehow grounded upon Tomas Elsaesser's "cinema effect" and the "cinematic perception [that] has become internalized as our mode of cognition and embodied experience." The necessity and abundancy of outdoor wireless hotspots and our perversely regulated culture by screen driven actions (walking in streets while watching a mobile device for example) validate this peculiar effect and perception.

Re-situating the argument of Elsaesser in our contemporary living patterns, we are at the threshold in which a broad range of disciplines - from architecture, design and the humanities to ICT and media. All call for acknowledge the limits of our ability to keep "thinking with thoughts" when there seems to be a pervasive replacement of our thoughts by digitally moving images. The German film theorist is thus suggesting tracing the multiple dynamics of this replacement and, in doing so, drawing on a range of new strategies and techniques. Indeed, during the 2010 s', new terms slip into the practice of enhancing places - digitization, multimedia, installation, interaction, interpretation, process-driven experiential strategies, and collective culture - to describe new abilities to steer pathways through open public space development and the individual participation to access, retrieve and interact with the wirelessly transmitted information which scarcely existed before the $1990 \mathrm{~s}$.

Today, there is a considerable number of research initiatives with similar and even more interactive initiatives working with the making of mediated public spaces. Many of them depend on the locomotional position and the active participation of the user, making them promising examples for the neo-analog scene that this book prefigured at the beginning. By just reading and exploring further some of them presented within the previous sessions one can argue that the battle for humanizing the postdigital outdoor landscape is about to be won. While most of them deal with the interaction between real and virtual, arguably the epitome of the "simulation" heritage, it is no surprise that within them the issue of the mediated place keeps blending seamlessly with the question how the physical space can employ the digital component to motivate, engage and inject the user to the hyperconnectivity of the information.

\footnotetext{
${ }^{1}$ Here quoted from Reimer H. (2009). Awaiting the voice-over: the Øresund Film Commission location database and the mediatization of architectural landscape. In Chaplin and Stara (Eds.). Curating Architecture and the City. Taylor \& Francis, p. 72.
} 
This fourth session does not intent to equate the computational turn in placemaking as the possibility for hybrid outdoor experiences with digital platforms and applications per se. This has already been done by the endless online projects that everyone with a mobile "on the go" connection can access and retrieve in many parks and squares. The turn heavily based on rapidly changing technologies - has already been shifted by time. The "cinema effect" is here understood as a critique to those digital modes of outdoor activities that revolve around the view that the offer of online information is the primary and user engagement with space is secondary. We can thus question the passivity of the search and retrieval powers of the "databases on the go", accepting a research into new dimensions formed from a neo-analog materiality. In this sense, the session attempts to inquire some creative ways in which the intellectual properties of the man - space relationship can reside within the structure of digitally enhanced experiences, even when traditional aspect of our outdoor living have been filtered and transferred to a projected immaterial state.

While outdoor human tasks are in a way even more pervasively regulated by machines, the humanistic content of the interaction often raises them in a more conceptual level. Cramer (2014), writing for A Peer Reviewed Journal About Post-Digital Research, described them as "neo-analog do-it-yourself" approaches that distant themselves from their "post-digital" predecessors that were simply referring "to a state in which the disruption brought upon by digital information technology has already occurred". Here, the term "neo-analog" doesn't refer to a chronological descendant of the post-digital, but to a shift that causes a new perspective to be emerged, one that expresses itself in complex patterns of user - machine interrelated activities, interlinked variables, and so forth. To some extent, we trace such intellectual/interactive tendencies in different neo-analogue methodologies and projects found around the web: from generative mindmaps, force-feedback algorithms to digital semantic relations, applications for visual music etc. Moving the issue of methodology to the foreground of a neo-analogue environment, offers us a great challenge to question the limits of hyperconnectivity in outdoor open public spaces.

This session situates the chapters within such a challenge and considers their innovative character to be a result not only of technological solutions but also of a humanistic re-orientation and re-focus. Having this in mind, it is rather critical to draw a distinction between two central aspects of our contemporary outdoor landscape given by man - machine convergence, a distinction according to which the digital can identify itself as a tool and as a of method.

The first aspect, in the sense of a "diagnostic" medium to access and investigate dimensions of the man/machine interaction, enables scholars to use the digital dimension in order to challenge the traditional boundaries that distinguish the spatial experience from the virtual one. Only the last decade, through transdisciplinary researches and explorations, we have come into possession of a challenging "functioning" tool with which places (and not only) have been enhanced and reactivated. From haptic or conversational interfaces of (some) embodied intelligence to platforms and networked systems of computers that capture and analyse inductive or deductive user responses, the idea of the digital as a medium tool to understand the importance of connectivity in different levels has significantly evolved the last decade. Yet it was only during the early-2010s that the idea of enhancing the already mediated public space received a distinctive typology: that of programming good public spaces by focusing 
"on the ability of digital technology to enhance communication and interaction with (potential) users, as a way to transform the production and uses of public spaces into an interactive process, enabling creative community participation and empowerment" (Smaniotto Costa 2017:19). To mention an example, the CyberParks Project (20142018) explored the typology of the mediated and hybrid outdoor place by investigating "the shape and scope of ICT impacts and the opportunities opened to improve the legibility and liveability of urban spaces, as well as new forms of integrating people's needs into urban design processes" (ibid:19). The second aspect of the method, in the sense of combining an intellectual inquiry into the digital landscape, refers to the cultivated logic beyond the tool and refers to user's agents of decision and choice.

\section{Digital Methods and Tools onto Hybrid Space}

The idea of the digital as a tool to increase the understanding of different levels of connectivity and to transform this knowledge into workings methods winged the discussions in Working Group 1 Digital Methods - of the CyberParks COST Action TU1306. The aim of this working group was to explore the digital as tool inserted into a research or methodology to increase the knowledge on people's use of public spaces (from current uses to future needs). This made the call to examine also user-behaviours and user's spatial needs. Within this working group scientific, technical and sociological information (or opportunities) were assessed, all relevant for understanding the interweaving of people in (and with) public spaces. Field experiments with new tools and methodologies were undertaken in several case studies.

The following chapters in Part IV, are based on the outcomes of the Working Group on Digital Methods. This working group had as main objectives: (1) to identify promising working approaches with ICT-tools and promote their use/test in different case studies; and (2) aggregate the experiences gained in the cases studies to a structure that help to better understand the ICT-tools available. For this an integrated framework could be useful, especially for aiding the development of people-friendly cities or the building of the communities' capacity to engage with their environment. The first step consisted on defining a classification rationale and a structure to specify the different uses and ways of penetration of technology into public spaces. This task was more easily said than done. First, the interactions between people and places were crucial for the CyberParks Project, and this interactions are per se a very large area of work, as demonstrated in the diversity of the topics tackled in this book. Second, this large area of work becomes even larger when the digital intertwines places and people relations. Fact is that there are several ways to tackle the pervasiveness of ICTs, which is intensified by the speed of technological development. Innovation is entering both the market and the city at an accelerated pace (Smaniotto Costa et al. 2017), challenging not only users but also those who want to understand their benefits in the medium and ling term. The Working Group on Digital Methods worked out three different frameworks - these are not mutually exclusive but can be used in complementary ways.

The first framework for an integrated approach resulted in a structure according to the degree of users' engagement along with the device/media/application used. 
The framework is displayed in form of a tree-structure that groups the identified ICTtechnologies into four main categories: Augmented Reality, Localization Technology, Wireless Network and Vision Technology. These four main dimensions are closely related to spatial quality, user needs, spatial attributes and added value delivered by the provision/implementation/availabily of ICT in public spaces. This framework backed the research design in the case studies developed within the Project. The results of case studies are described in several chapters of this book (Fig. 1).

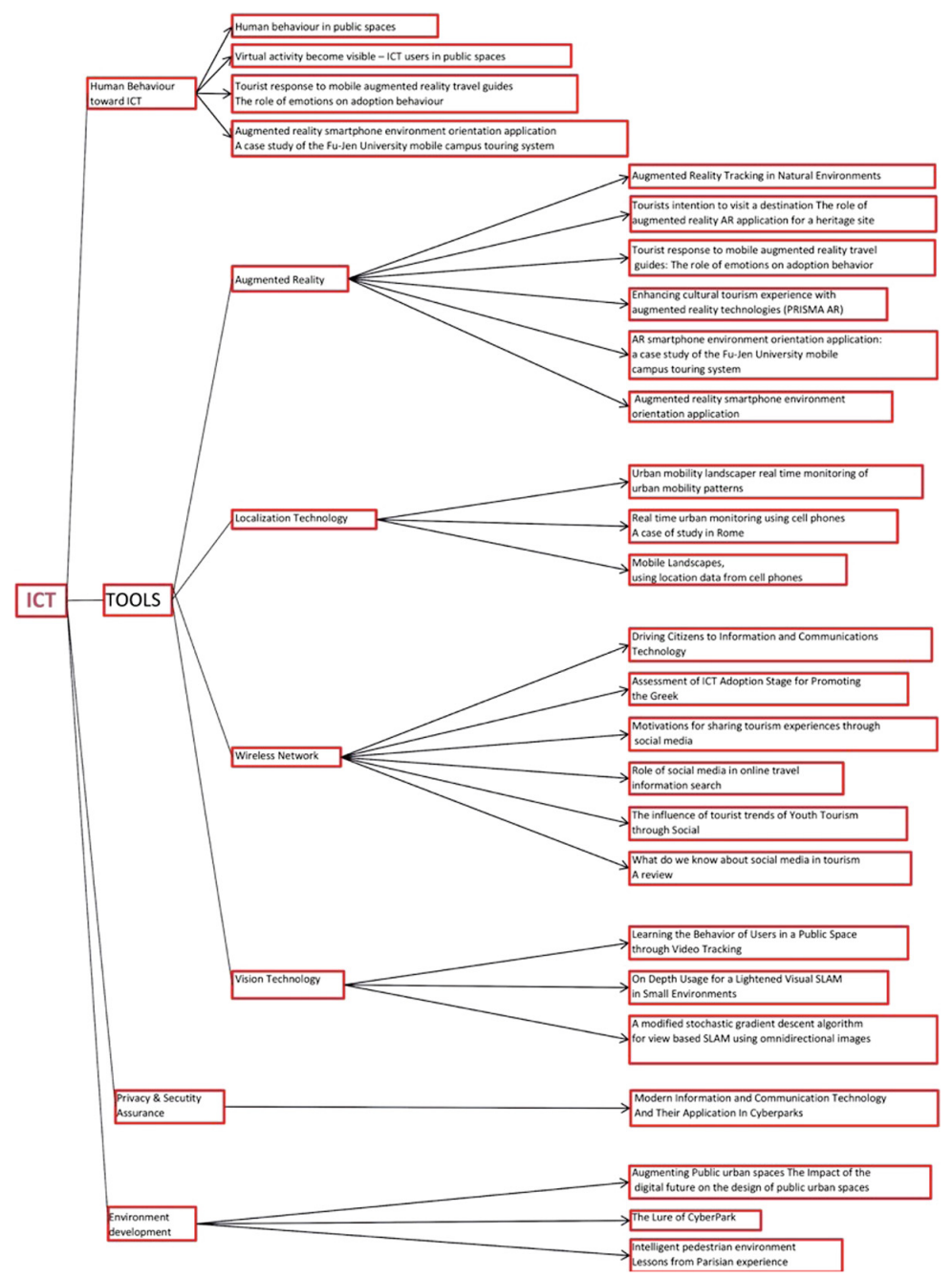

Fig. 1. Shows the main structure to understand the diffusion of the digital into public spaces

The second framework is based on the matrix of the applied technology and the added value this application results for users and uses of places. In this framework, the technology is understood in three categories: Position Informatics, Sensory informatics 
and Synergetic interfaces, when implemented in public spaces these technologies can result in several benefits (added values). The added values are listed as Enhance publicness, Increase the performance of public spaces, Increase the production and cocreation of public spaces, Increase the understanding on users, Increase the understanding on uses, Dissemination of information in/about public spaces, and the Use of ICT as a support and challenge for new outdoors activities. This framework is used in the POOL OF EXAMPLES ${ }^{2}$ the Project CyberParks established. The Pool offers a wide range of examples of the penetration of technology into public spaces. Based on the available technology (selected into the three categories), a response matrix was built with the types of public spaces. With the Pool CyberParks seeks to increase the understanding of the benefits of technology to enhance places in order to achieve an added value (i.e. new outdoor experiences, innovative ways of using places).

The third framework is built according to the purposes of the ICT into spaces. This can be primarily structured in three major dimensions: (1) for research, i.e. as a way to produce, collect, manage, mediate and interpret data, (2) for design, i.e. as a range of possibilities for conceiving and/or creating public spaces, and (3) for implementation, i.e. by looking onto the transformations of the material production of space and and/or social interaction triggered by the continuous introduction of new hardware and software (Fig. 2). Further insights on this framework can be obtained at Smaniotto Costa et al. (2017).

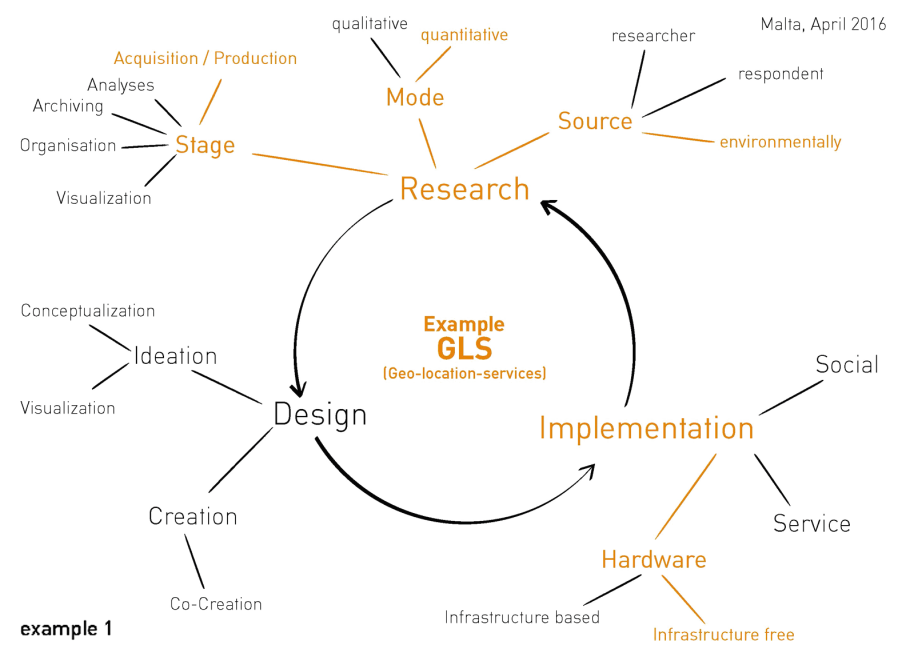

Fig. 2. Shows three modes of ICT use; highlighting the example of Geo-location services, it demonstrates the different subsystems or components, i.e. for research and implementation.

These three frameworks evidence the need to adopt integrated frameworks, those that enables a full overview on the benefits of technology advancements, and as noted above these are taking place at a rapid pace, challenging users, designers and

\footnotetext{
The Pool is available at www.cyberparks-project.eu/examples, and enables the searching, navigating and adding new examples.
} 
researchers. As highlighted in this introduction, public spaces remain a timeless patrimony and in Smaniotto Costa et al. (2017:172) about the quality of places, which [...] remains a central issue, even in the digital era. No one will leave their home and use a public space, if it isn't safe or doesn't offer the requirements. Quality public spaces make up the richness of urban life."

\section{Overview of Chapters}

Acknowledging that digital hyperconnectivity usually offers the norms that affect people's behaviour and decisions, we can embark on a much larger investigation that touches fields that are heavily defined by humanities such as response, education or cognitive development. In this sense, and although interrelated, the "method" can be conceptually separated from the "tool" and it is in this sense that some chapters of this session present their working hypothesis.

In the first chapter (4.2) of part IV, Barbora Čakovská, Mária Bihuňová, Preben Hansen, Ernesto Marcheggiani and Andrea Galli in Methodological Approaches to Reflect on the Relationships Between People, Spaces, Technologies describe different methodologies, backed by their rationales. The authors are convinced that the ICT advancements can lead to increase in attractiveness of public spaces for both citizens and visitors. Moving in and through the space and understanding its features is still an important human activity, and as such it is susceptible to changes. In the blended space, so the authors, designers need to create and establish interfaces and systems that enable people to shape and achieve their goals and aspirations. These interfaces should include and inherit different physical, digital, perceptual, sensory and conceptual point of contact that are between people and the content that is contained and accessible by.

Following this, in chapter 4.3 In Modelling co-creation ecosystem for public open spaces, Aelita Skarzauskiene, Monika Maciuliene and Petja IvanovaRadovanova argue that the logic of ecosystem presents a valid template for a tentative method toward the exploration of the new context and relationships between people, places and technology. The dynamics in the co-creative ecosystem propose an alternative reading of embedded networks based on the process of value creation. Inquiring the role (of actors) becomes a method by which to dislocate aspects of the network in a manner through which we can rethink "who can offer value in space". Their model suggests a method of approaching the value as emerging out of " $a$ number of entities [that] work collectively to create mutual benefits by granting access to one another's resources including people, technologies, organizations and information".

In chapter 4.4 Eneko Osaba, Roberto Pierdicca, Tiago Duarte, Alfonso Bahillo and Diogo Mateus in the work entitled Using ICTs for the Improvement of Public Open Spaces: The Opportunity offered by CyberParks Digital Tools, introduce the three tools developed by the CyberParks teams: WAY CyberParks, CyberCardeto and EthnoAlly. The functionalities and features of each tool are described and discussed on the basis of different case studies. Backed by different technology and developed with different purposes, the three tools open for an interaction between researchers, city councils and users an innovative and wide range of possibilities. They offer valuable 
resources to make a significant contribution to the study of public spaces, enabling a quick and efficient collection of data, essential for a better understanding the interactions of people with the spaces. On the flip side, the tools allow users to get dynamic contextual information related to space, and therefore a better knowledge about their surroundings. The common feature of the three tools are free to use across the world.

To meet the posed challenge to better understand the benefits of mediated places, the chapter 4.5 by Bonanno, Klichowski and Lister: A Pedagogical Model for CyberParks, attempts to redefine the field of pedagogy as a composite neo-analogue experience involving intra-individual cognitive, affective and conative interactions together with inter-individual, networked, interactional processes. In their contribution, they theorise the "society of mediated learning" with digital tools while on the move and present a methodological insistence on "learning by combining two types of cerebral operations: motor and cognitive control". The authors further contend that the hybrid environments may trigger deep learning, this in turn changes one's competence profile and epistemological conception. Cyberpark, the mediated place, challenge people, so the authors: to extend their learning boundaries through acquisition of new knowledge and skills, by sharing their understanding and by contributing to the distributed knowledge and networked experience.

The final chapter 4.6 by Jamal Raiyn and Jugoslav Jokovic, entitled The application of advanced IoT in public spaces towards promote their safer use, examines how IoT can be used to manage the resources in hybrid places. The central issue of the authors is to clarify the opportunities of IoT tools in public spaces to prevent crime and to increase the safety of users. Their analysis contributes to a broader discussion towards their primarily interest: developing a new scheme for securing the privacy of users in a mediated public space.

Methodological aspects of digital mediacy in public open spaces have become part not only of our digital culture but also of our own evolved mechanisms of thinking and understanding space and place. The rising number of online cutting-edge technologies that undertake the task of dislocating outdoor experience from the analogue space of coordinate relations to the digital landscape and virtual reality of algorithmic relations is a testament to the distinction between using the digital as a "tool" and as a "method". Grasping the opportunities that the mediated space open is a central issue in the chapters of Čakovská et al. (4.2) and Osaba et al. (4.4), and Raiyn and Jokovic (4.6) to some extent. In their arguments the authors place emphasis on ICT as a tool and as method to increase knowledge through data analysis - with different tools and through different methods - the relational aspects between the physical places and users. Undoubtedly, the analogue space of urban squares and green parks remains a timeless patrimony, a constitutive dimension that deeply embeds itself to our cities as an engenderment of the materiality and physicality of urban form. It is also the traditional medium by which the logic of its symbolic systems and intellectual combinatory in the poetic world of Bachelard (1994) are transformed into material variations, allowing thus the reasoning human mind be attached to the sensible world. The analogue is the forefather; that necessary element which public space is tied up with in order to lend itself to further dialectical investigations that extend beyond the practical function of its forms. Čakovská et al. (Chapter 4.2) argue further that space is largely build by society and it makes no sense to conceive space without a social content, just as society does not exist 
without a spatial component. This interdependency, in a continuous process, creates and modifies spaces, while such transformed space at the same time influences the society. It is the common starting point from which we are infused in even more complex conceptions of either geometric or symbolic order - to mention one example, Arnheim's (2004) symbolic readings of forms seized upon the field of the analogue and its visual qualities as a way to project them "as images of the human condition" instead. The process and context dimensions of the network, as articulated in Skarzauskiene, Maciuliene and Ivanova-Radovanova's view (chapter 4.3), epitomize the heterogeneity of the co-creative dimension, essential for the understanding of the humanist content of the proposed ecosystem.

However, the chapters of this part argue that in the contemporary digitally mediated landscape in which the materiality and physicality of things give place to the impression drawn from protocol-based representations (computational images, animations or texts), we impose upon our conception of space a topological dislocation technique that has, quite literally, a reversible logic. In an interesting way, and from Arnheim's perception-and-response to the analogue stimuli evolving conception, the (post)digital dimension brings in a significant effect on this traditional process retaining at the same time its humanist condition - that is the active and personal aspects found within. The displacement, reacting to the withdrawal of the analogue materiality, seems to inaugurate a reversed method to understand man - machine interaction: from the observer's responses to data elements, the mind is challenged to construct an a posteriori perceptual content in the imaginary register, or better a trace to use a laconic term. This content is not as deterministic as its precedent analogous one, but can resurface in alternative versions based on an exchange of conscious and unconscious thought. However, is this trace enough to speak about a digitally-inspired method beyond the functioning tool and any technological underpinnings of the displacement? The chapters of this session suggest that the answer to this question is not an easy one while more complicated phenomena may appear. The digital mediacy doesn't only refer to "functional" aspects like information visualization and its presentation as a collection of online material. As argued by Bonanno, Klichowski and Lister (chapter 4.5), it goes into the transposition of deeper parts of the outdoor activity that take place in human mind, in desire, in mental patterns and conscious or unconscious organizational mechanisms. These are the deeper aspects that traditionally bound spatial experience with the subject/object relation. They are responsible for the possibility of the mind, by the use of the digital dimension, to implicate in transformational processes from abstract and intelligible thought to enhanced perceived relations. And in a sense, these are the main aspects from within mediated places enact as a dialectic activity between subject and object and begin to exist as a reflecting method outside the physical or Euclidean space.

The shift from Tool to Method is the departure point to discuss this apparent difficulty and the oxymoron that brings in: the digitalization and dislocation of experience that is meant to be analogue. This session is not at all about "mere digitization" and online dissemination of information. It is about how to turn, momentarily at least, a functional tool into a method by relating human mental activities like learning, desire, meaning and reason acquisition to an artificial intelligence. 
In reading the following chapters, we have to say, the first reflex is to dismiss the concept of a "neo-age" from a nostalgic and mimetic revival of stereotyped ways of experiencing the contemporary public space, one that abolish space's valuable heritage from its injection with the digital technologies. It is an objective fact that the emerging methodologies discussed within the following chapters approach the space-related data as being "digital" almost by definition. Data to be collected, sorted, shared and reused across dynamically established links between analogue (human tasks) and digital (computational executions) methods of thinking and acting. In the age of the semiotic web and the web 3.0, these modes are nowadays beyond interactivity. They are intelligent forms of studying information based, for example, on users' personal desires and aspirations while utilising probabilistic parameters or algorithmic decision-making. As the chapters discuss later on, the neo-analogue methodologies presented from within the following pages propose a hybrid attitude preserving at the same time the analogue and symbolic value of open public space within digital actions.

\section{References}

Arnheim, R.: Art and Visual Perception: A Psychology of the Creative Eye. University of California Press, Berkeley (2004)

Bachelard, G.: The Poetics of Space. Beacon Press, Boston (1994)

Cramer, F.: What is 'Post-digital'? A Peer Reviewed Journal About Post-Digital Research (2014). http://www.aprja.net/?p=1318. Accessed 03 Sep 2014

Mitchell, W.: Me++: The Cyborg Self and the Networked City, p. 58. MIT Press, Cambridge (2004)

Smaniotto Costa, C.: A framework for defining principles for inclusive mediated public open spaces. In: Smaniotto Costa, C., Ioannidis, K. (eds.) The Making of the Mediated Public Space: Essays on Emerging Urban Phenomena, pp. 17-24. Lusófona University Press, Lisbon (2017)

Smaniotto Costa, C., Ioannidis, K. (eds.): The Making of the Mediated Public Space: Essays on Emerging Urban Phenomena. Lusófona University Press, Lisbon, Portugal (2017)

Winston, A.: "Scary" Venice Architecture Biennale show has "nothing to do with design" says Koolhaas (2014). www.dezeen.com/2014/06/05/rem-koolhaas-venice-architecture-biennale2014. Accessed 22 Nov 2018

Open Access This chapter is licensed under the terms of the Creative Commons Attribution 4.0 International License (http://creativecommons.org/licenses/by/4.0/), which permits use, sharing, adaptation, distribution and reproduction in any medium or format, as long as you give appropriate credit to the original author(s) and the source, provide a link to the Creative Commons license and indicate if changes were made.

The images or other third party material in this chapter are included in the chapter's Creative Commons license, unless indicated otherwise in a credit line to the material. If material is not included in the chapter's Creative Commons license and your intended use is not permitted by statutory regulation or exceeds the permitted use, you will need to obtain permission directly from the copyright holder.

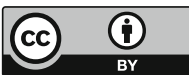

